\title{
Androgen receptor promotes oral squamous cell carcinoma cell migration by increasing EGFR phosphorylation
}

This article was published in the following Dove Press journal: OncoTargets and Therapy

\author{
Xin Liu' \\ Shanglan Qing ${ }^{2}$ \\ Keke Che ${ }^{3}$ \\ Lihua $\mathrm{Li}^{4}$ \\ Xiaoming Liao ${ }^{5}$ \\ 'Department of General Dentistry, \\ Chongqing Savaid Stomatology Hospital, \\ University of Chinese Academy of \\ Sciences, Chongqing 400014, People's \\ Republic of China; ${ }^{2}$ Department of \\ Stomatology, Chongqing General \\ Hospital, University of Chinese Academy \\ of Sciences, Chongqing 4000I4, People's \\ Republic of China; ${ }^{3}$ Department of \\ Pharmacology, Chongqing General \\ Hospital, University of Chinese Academy \\ of Sciences, Chongqing 4000I4, People's \\ Republic of China; ${ }^{4}$ Department of \\ Stomatology, Affiliated Hospital of North \\ Sichuan Medical College, Nanchong, \\ Sichuan 637000, People's Republic of \\ China; ${ }^{5}$ Department of Stomatology, \\ Chongqing Prevention and Treatment \\ Hospital for Occupational Diseases, \\ Chongqing 400060, People's Republic of \\ China
}

Correspondence: Shanglan Qing Department of Stomatology, Chongqing General Hospital, University of Chinese Academy of Sciences, 104 Pibashan Main Street, Chongqing 4000I4, People's Republic of China

Email ares_young@I63.com
Objectives: This study is aimed to investigate the role of androgen receptor (AR) in regulating oral squamous cell carcinoma (OSCC) cells migration.

Materials and methods: Tumors from 23 patients with OSCC and five OSCC cell lines were used for analyzing AR expression. The effects of AR agonist and antagonist were used to examine the role of AR in regulating the migration of OSCC cells.

Results: Ten of 23 tumors from patients with OSCC were AR positive. There was no significant difference in total EGFR (tEGFR) expression between AR-positive tumors and AR-negative tumors. However, the expression of phosphorylated EGFR (pEGFR) in ARpositive tumors was significantly higher than that in AR-negative tumors $(p<0.01)$. Stimulation of AR by dihydrotestosterone in SCC9 (AR-positive OSCC cell) caused an increase in pEGFR and pAKT expression and promoted cell migration without changed tEGFR expression, whereas treatment with bicalutamide led to a decrement in pEGFR expression and pAKT and inhibited cell migration. No effects were found in SCC25 cell line (AR-negative) either treated by dihydrotestosterone or bicalutamide. Furthermore, SCC9 cell line treated by EGF or cetuximab (EGFR inhibitor) significantly promoted or inhibited cell migration.

Conclusion: Our data indicate that OSSC tumors and OSCC cell lines express AR which is critical for promoting cell migration by increasing EGFR phosphorylation.

Keywords: oral squamous cell carcinoma, androgen receptor, EGFR, migration, phosphorylation

\section{Introduction}

Oral squamous cell carcinoma (OSCC) is a malignant tumor with a high incidence and recurrence rate worldwide and accounts for over $90 \%$ of oral cancers, ${ }^{1}$ with an annual worldwide incidence of over 300,000 cases and a mortality rate of $48 \%{ }^{2}$ Two-thirds of OSCC occurred in men. ${ }^{3}$ At the early stage, the standard treatment is effective for patients with OSCC; however, eventually, more than $50 \%$ of patients develop local recurrence or distant metastases, resulting in a poor prognosis., ${ }^{4,5}$ Therefore, development of novel therapeutic strategies for patients with advanced OSCC, and further elucidation of the molecular mechanism that promotes the malignancy of OSCC, are urgently needed.

One feature of OSCC is progressive local invasion. ${ }^{6}$ To improve the treatments for OSCC, it is important to investigate its underlying invasion mechanisms. The processes involved in cancer invasion include cell migration, an external stimulus that 
affects the invading cells and interaction between the tumor and stroma at the invasive front, and the involvement of growth factors. ${ }^{7,8}$ To facilitate the identification of novel therapeutic targets, it is necessary to elucidate the signaling mechanisms underlying the regulation of cell migration. ${ }^{9,10}$

The androgen receptor (AR), a ligand-dependent transcription factor, belongs to the nuclear receptor superfamily. AR activated by androgen dimerizes as a homodimer and subsequently binds to androgen-responsive elements (AREs) on the promoter regions of target genes. This binding activates the expression of genes regulating the growth, differentiation, and survival of AR-expressing cells, such as prostate epithelial cells. ${ }^{11}$ AR signaling is critical in carcinogenesis and the development of prostate cancer $(\mathrm{PCa})$, and for patients with advanced prostate cancer, blockage of AR signaling has been used as a standard treatment. At present, there is limited evidence of the expression and function of AR in OSCC tumors compared with the intensive studies for functions of $\mathrm{AR}$ in prostate cancer. ${ }^{12}$

Epidermal growth factor receptor (EGFR) is one of the four members (EGFR, HER2, HER3, and HER4) of the transmembrane ErbB family. EGFR plays a critical role in the signal transduction pathways that regulate cellular function, including proliferation, migration, differentiation, and regulation of anabolism in tumor cells. ${ }^{13,14}$ Targeting EGFR has been used as a standard of care in lung cancer and colorectal cancer for selected patient populations. ${ }^{15,16}$ EGFR has been found to be upregulated and overexpressed in the majority of oral cancers and is associated with a poor clinical prognosis. ${ }^{17}$

It has been shown that AR expression promotes EGFR activation, which involves protein complex formation between AR and EGFR. ${ }^{18}$ AR activity also enhances the phosphorylation of EGFR and its downstream targets in breast and prostate cancer cells. ${ }^{19,20}$ A few studies have reported AR expression in OSCC and its function in the proliferation of OSCC. However, no studies were found to investigate the role of AR in the migration of OSCC cells. In this present study, we examined the correlation of the expression of AR, EGFR, and pEGFR in OSCC tumors and identified AR expression in OSCC cell lines. We further investigated the function of $\mathrm{AR}$ in promoting the migration of OSCC cells by increasing EGFR signaling.

\section{Materials and methods}

\section{Tumor tissues and immunohistochemistry}

In this study, 23 patients with OSCC, not previously treated with chemotherapy and/or radiotherapy, were consecutively recruited. All patients with a mean age of 52.9 years (range 31-68 years) were otherwise healthy; 15 were male and 8 female. Patient samples were collected with written informed consent in accordance with the guidelines of the Declaration of Helsinki. This study was approved by the local Ethics Committee of Chongqing General Hospital, and all subjects gave written informed consent prior to participation.

Paraffin sections were deparaffinized in xylene, rehydrated through graded alcohol, and processed for antigen retrieval by in microwave preheated $10 \mathrm{mM}$ citrate buffer (pH 6.0) for $15 \mathrm{~min}$. Sections were incubated in 3\% H2O2 in $50 \%$ methanol for $10 \mathrm{~min}$ at room temperature to quench endogenous peroxidase. To block nonspecific binding, sections were incubated in 5\% BSA for $30 \mathrm{~min}$ and then a biotin blocking system (Dako Denmark A/S, Glostrup, Denmark) was used to block endogenous biotin. Sections were then incubated with anti-AR (1:100; Abcam, Cambridge, UK, ab9474), anti-EGFR (1:100; Santa Cruz Biotechnology Inc., Dallas, TX, USA, sc373746), or anti-pEGFR (1:100, Abcam, ab40815), at $4^{\circ} \mathrm{C}$ overnight. After rinsing, sections were incubated with HRP polymer kit (Biocare Medical, Pacheco, CA, USA; GHP516) for $60 \mathrm{~min}$ at room temperature, followed by 3,3-diaminobenzidine tetrahydrochloride as the chromogen. Then the slides were counterstained with hematoxylin and mounted after dehydration.

\section{Cell lines}

OSCC cell lines (SAS, SCC4, SCC9, OECM-1, SCC25) and human prostate cancer cell lines (LNCaP) were purchased from American Type Culture Collection (ATCC) (Manassas, VA, USA) and maintained in RPMI 1640 (Wisent, Nanjing, People's Republic of China) containing 10\% FBS (ExCell Bio, Inc., Shanghai, People's Republic of China), penicillin $(100 \mathrm{U} / \mathrm{mL})$ and streptomycin $(100 \mathrm{mg} / \mathrm{mL})$. All cell lines were cultured in a humidified air atmosphere containing 5\% $\mathrm{CO} 2$ at $37^{\circ} \mathrm{C}$.

\section{Migration assay}

Cell migration was evaluated by using a scratch wound healing assay as previously reported. ${ }^{21}$ Cells were seeded in 24well plates at a density of $10^{4}$ cells/well in complete DMEM and cultured to confluence. The cell monolayer was serum starved overnight in DMEM prior to initiating of the experiment. To block cell proliferation, cells were treated by $10 \mu \mathrm{g} /$ $\mathrm{mL}$ mitomycin $\mathrm{C}$ for $4 \mathrm{~h}$. The confluent cell monolayer was scraped with a pipette tip to generate scratch wounds. The cells were then washed with PBS and an image was captured using 
a Nikon Eclipse TE2000-5 microscope (Nikon Corporation, Tokyo, Japan) to record the wound width at $0 \mathrm{~h}$. Cells were incubated at $37^{\circ} \mathrm{C}$ for $20 \mathrm{~h}$ with the conditioned media containing either dihydrotestosterone $(10 \mathrm{nM})$, or bicalutamide $(10$ $\mu \mathrm{M})$, or EGF $(20 \mathrm{ng} / \mathrm{mL})$, or cetuximab $(20 \mu \mathrm{g} / \mathrm{mL})$, or null control. In order to evaluate the rate of migration, images were captured again after incubations for $20 \mathrm{~h}$.

\section{Quantitative RT-PCR}

Total RNA was extracted from the cultured cells using RNeasy kit (Invitrogen, St Louis, MO, USA), according to the manufacturer's protocol. RNA samples were subjected to reverse transcription using a PrimeScript RT Reagent Kit (Takara, Dalian, People's Republic of China). The reactions were run in triplicate in three independent experiments. Data were collected and analyzed using the Rotorgene software accompanying the PCR machine. The CT values for the samples were normalized to the corresponding GAPDH CT values, and relative expression levels were calculated using the $\Delta \Delta \mathrm{CT}$ method.

\section{Western blot}

Western blot analysis was performed as follows. Proteins from isolated cells were electrophoresed under reducing conditions in $10 \%$ SDS-PAGE gels and transferred to nitrocellulose membranes. The blots were blocked in 5\% milk, incubated overnight at $4^{\circ} \mathrm{C}$ with the primary antibody (anti-AR $(1: 1,000$, Abcam, ab9474)), anti-EGFR (1:1,000, Santa Cruz Biotechnology, sc-373746), anti-pEGFR (1:1,000, Abcam, ab40815), anti-AKT (Santa Cruz Biotechnology, sc-5298), and anti-pAKT (1:1,000, Abcam, ab81283), and incubated with secondary antibody for $1 \mathrm{~h}$ at room temperature. The specific signals and the corresponding band intensities were evaluated using an Odyssey Infrared Imaging system and software (LI-COR Bioscience, Lincoln, NE, USA).

\section{Statistical analysis}

All data are presented as the mean \pm standard error. To determine the significant differences between the groups, unpaired Student's $t$-test was used. $p<0.05$ was considered to indicate a statistical difference.

\section{Results}

AR-positive tumors expressed higher pEGFR without changes of tEGFR

Previous studies have reported that AR expressed in OSCC tumors. In this study, to evaluate AR expression in tumors from 23 patients with OSCC, AR immunohistochemical staining was performed. AR was mostly stained in the nuclei of cancer cells. Then, tumors (7 males and 3 females) were AR-positive (Figure 1A). Another 13 tumors were AR-negative (Figure 1B). A prostate tumor was used as a positive control (Figure 1C). EGFR expressed on the cellular plasma membrane controls its downstream signaling pathways and related functions. In all 23 tumors, tEGFR and pEGFR were nicely stained on the cell membrane (Figure 1D, E, G, and H). No difference in tEGFR expression was found between AR-positive tumors and AR-negative tumors (Figure 1F). In ARpositive tumors, pEGFR expression was significantly higher $(p<0.01)$ than that in AR-negative tumors (Figure 1I).

\section{AR expression in OSCC cell lines}

The expression of AR in different OSCC cell lines was examined. Prostate cancer LNCaP cells (AR-positive) was used as a positive control. As shown in Figure 2A, four OSCC cell lines (SAS, SCC4, SCC9, and OECM-1) expressed different levels of AR mRNA, which were lower than that in LNCaP cells. AR mRNA in SCC25 was not detectable. Different levels of AR proteins were expressed in the four AR mRNA detectable OSCC cell lines (Figure 2B). Compared to the $\mathrm{AR}$ expression in the LNCaP cells, AR protein in OSCC cells was much lower. AR protein was not detected in SCC25. SCC9 expressed the highest levels of AR mRNA and protein and SCC25 did not express AR, so SCC9 and SCC25 were used for the following studies.

\section{DHT promoted cell migration through increasing $P E G F R$ and $p A K T$ expression}

To determine the role of the androgen receptor (AR) in regulating oral squamous cell carcinoma (OSCC) cells migration. SCC9 (AR-positive) and SCC25 (AR-negative) were treated with AR ligand (DHT). DHT treatment increased SCC9 migration rate approximately 1.9-fold (Figure 3A, B, E, F, and I). In contrast, DHT treatment did not change the SCC9 migration rate (Figure 3C, D, and $\mathrm{G}-\mathrm{I}$ ). Compared with the vehicle-treated cells, treatment with DHT in SCC9 cells led to an increased expression of pEGFR and pAKT proteins with no changing of tEGFR mRNA and protein (Figure 3J and K). Of course, treatment with DHT in SCC25 did not cause any change of tEGFR, pEGFR and pAKT (Figure 3J and K). 

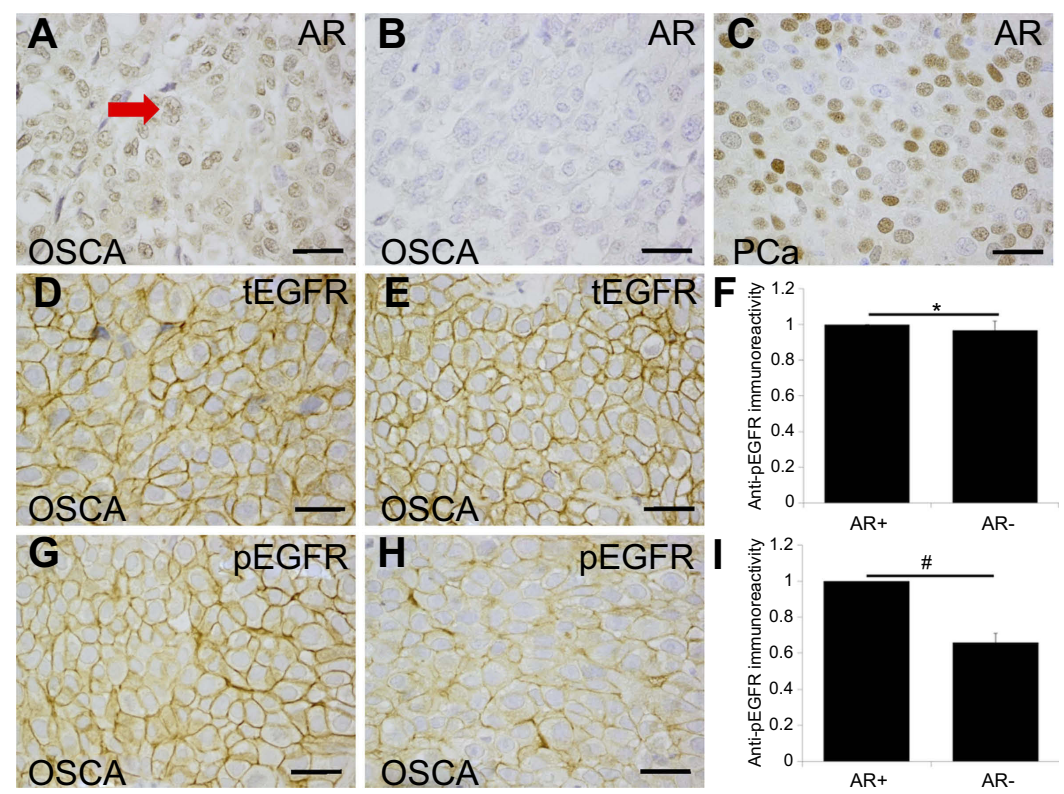

Figure I Expression of AR, EGFR, and pEGFR in OSCC tumors. (A) AR-positive staining in OSCC tumor (red arrow: cancer cell). (B) AR-positive staining in OSCC tumor. (C) AR staining in prostate cancer. (D and E) tEGFR staining in AR-positive OSCC tumor and AR-negative OSCC tumor. (F) No difference in tEGFR expression between AR-positive tumors and AR-positive tumors $\left({ }^{*} p>0.05\right)$. ( $\mathbf{G}$ and $\left.\mathbf{H}\right)$ PEGFR staining in AR-positive OSCC tumor and AR-negative OSCC tumor. (I) AR-positive tumors express significantly higher pEGFR ( $\left.{ }^{\#} p<0.0 \mathrm{I}\right)$. Scale bar in $\mathbf{A}-\mathbf{E}, \mathbf{G}$, and $\mathbf{H}, 50 \mu \mathrm{m}$.

Abbreviations: AR, androgen receptor; OSCC, oral squamous cell carcinoma; EGFR, epidermal growth factor receptor; pEGFR, phosphorylated EGFR; tEGFR, total EGFR.

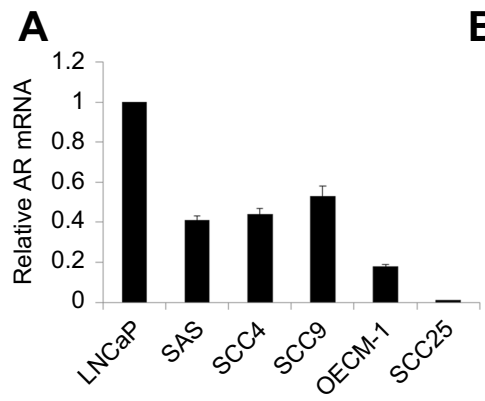

B

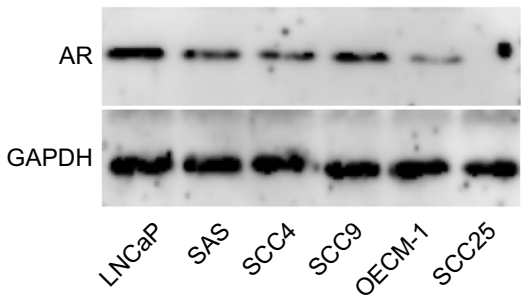

Figure 2 Expression of AR in different OSCC cell lines and LNCaP cells. (A) AR mRNA in LNCaP was used as a positive control, AR mRNA was detected in four OSCC cell lines, not SCC25. (B) Different levels of AR proteins expressed in these cell lines. Abbreviations: AR, androgen receptor; OSCC, oral squamous cell carcinoma.

\section{Bicalutamide inhibited cell migration through reducing $\mathrm{PEGFR}$ and $\mathrm{pAKT}$ expression}

To confirm AR signaling promotes migration of oral squamous cell carcinoma (OSCC) cells, SCC9 and SCC25 were further treated with Bicalutamide (an AR inhibitor). Bicalutamide significantly decreased SCC9 migration rate (Figure 4A, B, E, F, and I). On the other hand, there was no change in migration rate in SCC9 treated with Bicalutamide (Figure 4C, D, and G-I). Treatment with Bicalutamide in SCC9 cells decreased the expression of pEGFR and pAKT without changing EGFR mRNA and tEGFR protein in comparison with the vehicle-treated cells (Figure $4 \mathrm{~J}$ and $\mathrm{K}$ ).
Bicalutamide did not cause any change of tEGFR, pEGFR and pAKT in SCC25 (Figure 4J and K).

\section{EGF promoted, and cetuximab inhibited SCC9 cell migration}

To further investigate whether the effect of AR in SCC9 cells is through targeting EGFR signaling, SCC9 cells were treated by EGF or cetuximab. EGF treatment increased SCC9 migration rate approximately 1.7-fold (Figure 5A, B, E, F, and I). In contrast, the migration rate of SCC9 treated with cetuximab was suppressed by about 45\% (Figure 5C, D, and G-I). 


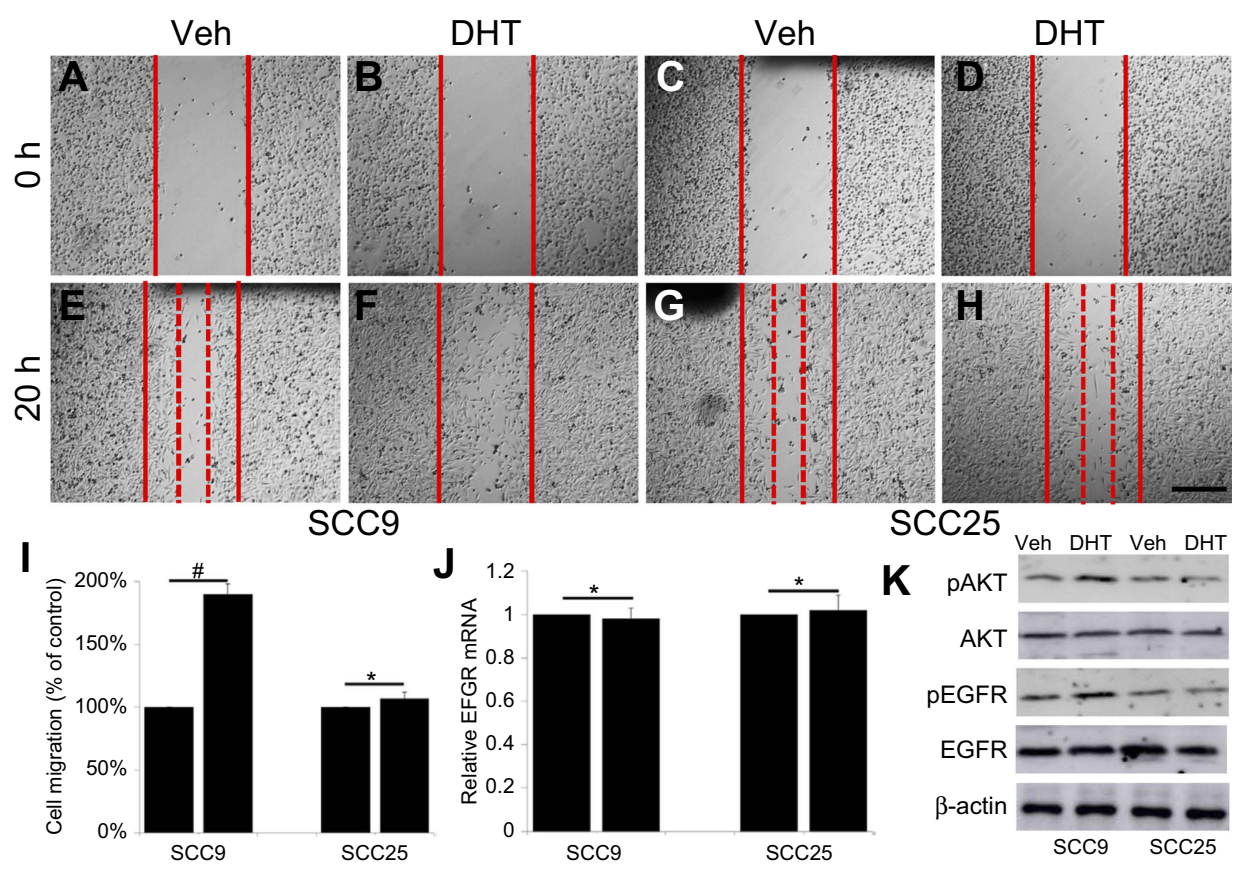

Figure 3 AR ligand (DHT) promoted the migration of SCC9 cells via increasing EGFR phosphorylation. (A, B, E, F, and I) DHT increased the migration of SCC9 cells following treatment with DHT for $20 \mathrm{~h}$. (C, D, and $\mathbf{G}-\mathbf{I})$ DHT did not change the migration of SCC25 cells. (J and K) In SCC9 cells, DHT increased expression of pEGFR and pAKT proteins with no changing of tEGFR mRNA and protein. DHT did not cause any change of tEGFR, pEGFR and pAKT in SCC25 (scale bar, $500 \mu \mathrm{m}$ ). Abbreviations: AR, androgen; EGFR, epidermal growth factor receptor; pEGFR, phosphorylated EGFR; tEGFR, total EGFR.

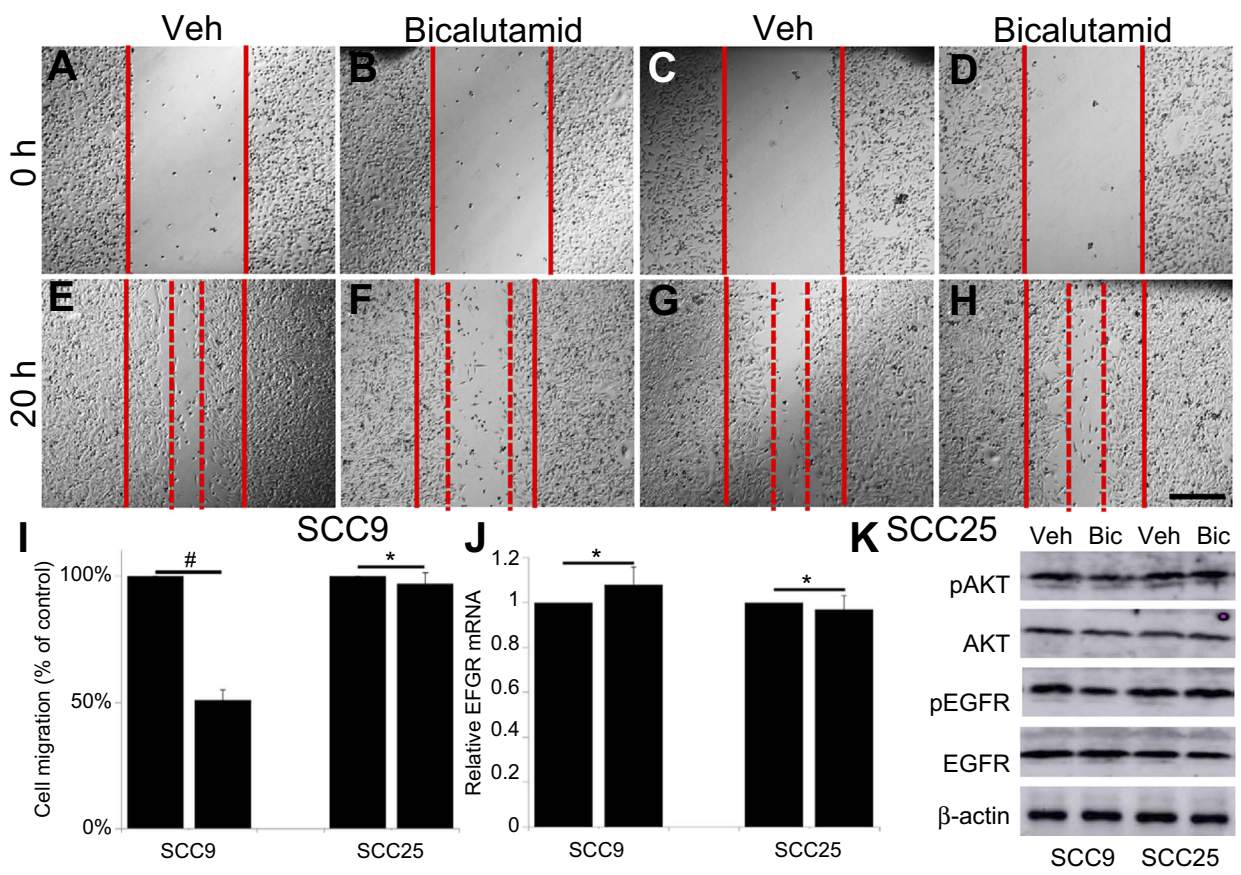

Figure 4 AR inhibitor (bicalutamide) inhibited the migration of SCC9 cells via decreasing EGFR phosphorylation. (A, B, E, F, and I) Bicalutamide decreased the migration of SCC9 cells following treatment for $20 \mathrm{~h}$. (C, D, and G-I) Bicalutamide did not change the migration of SCC25 cells. (J and K) In SCC9 cells, bicalutamide decreased expression of pEGFR and PAKT proteins with no changing of tEGFR mRNA and protein. Bicalutamide did not cause any change of tEGFR, pEGFR and pAKT in SCC25 (scale bar, $500 \mu \mathrm{m})$.

Abbreviations: AR, androgen receptor; EGFR, epidermal growth factor receptor; pEGFR, phosphorylated EGFR; tEGFR, total EGFR. 

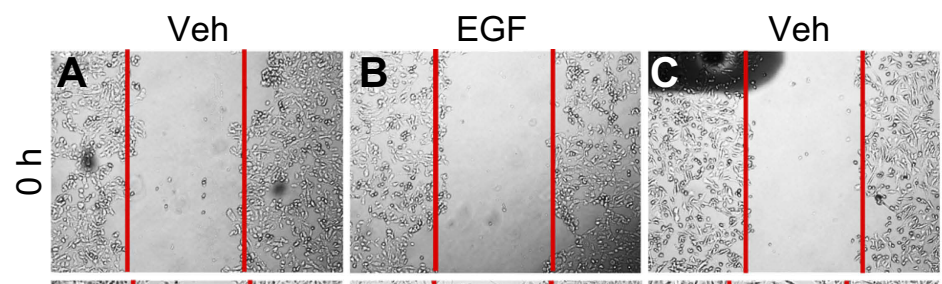

Cetuximab
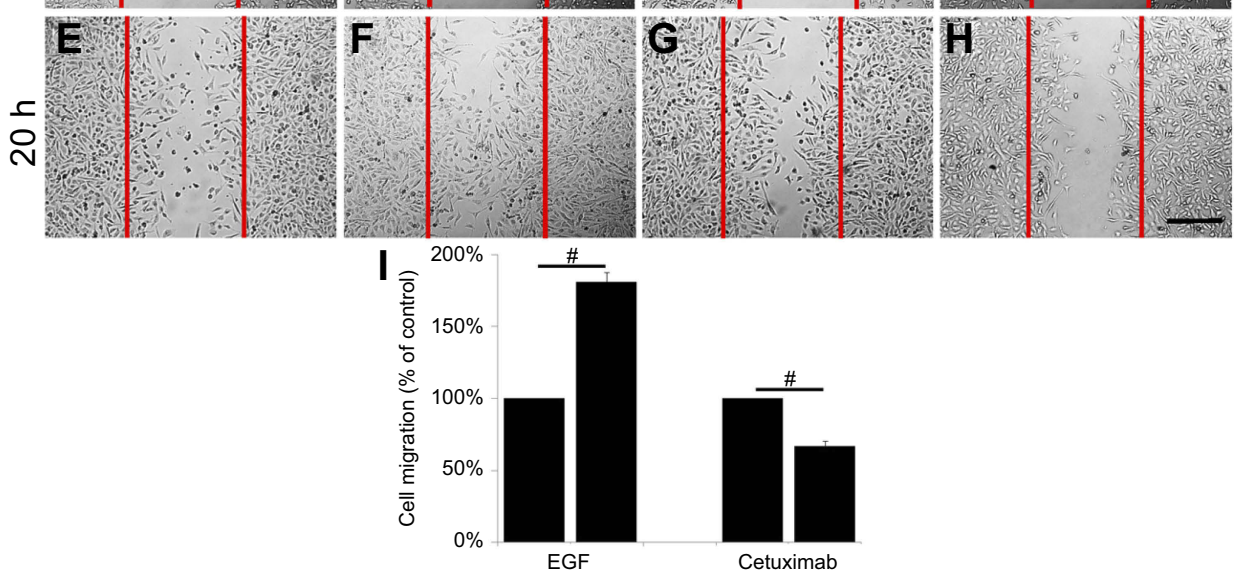

Figure 5 EGF promoted and cetuximab inhibited SCC9 migration. (A, B, E, F, and I) EGF increased the migration of SCC9 cells following treatment for $20 \mathrm{~h}$. (C, D, and G-I) Cetuximab decreased the migration of SCC9 cells following treatment for $20 \mathrm{~h}$ (scale bar, $500 \mu \mathrm{m}$ ).

\section{Discussion}

In this study, we first examined whether AR is expressed in OSCC samples and cell lines. The results confirmed that AR was expressed in 10 of 23 OSCC tumors (43.48\%). AR expression was positively correlated to $\mathrm{pEGFR}$ expression in OSCC tumors. Four OSCC cell lines expressed AR except for SCC25. The findings of this study showed that AR is critical for promoting the migration of OSCC cells by up-regulating EGFR phosphorylation. The studies provide new insights into the role of AR in OSCC cells migration.

In humans, AR ubiquitously expressing throughout the entire body exerts its effects on cells through both classical genomic mechanisms and rapid non-genomic actions. AR acts as a ligand-dependent transcription factor via a classical genomic mechanism which involves homo-dimerization and translocation to the nucleus upon binding androgen hormones, binding to specific DNA sequences, and recruiting co-regulators to initiate transcriptional changes over time. ${ }^{22}$ Dysregulated AR expression or activity is a key factor that causes the malignant transformation of prostate cancer cells, and thus, inhibition of AR activity is a therapeutic strategy to treat prostate cancer. ${ }^{23}$ It has been reported that AR was expressed in OSCC tumors. ${ }^{12,24}$ Our data clearly showed AR expression in $43.48 \%$ OSCC tumors, which was similar to the previous reports. Previous studies have shown that most of the AR expression in premalignant and OSCC tissues was in the cytoplasm, differing from the nuclear AR expression observed in prostate carcinomas. A study of 113 clinical specimens of non-prostate adenocarcinoma also revealed that 87 cases were AR-positive, and 66 (75.8\%) of these were cytoplasmic positive only. ${ }^{25}$ In this study, we found most of the AR expression was in nuclei of OSCC cancer cells, with little expression in cytoplasm. To confirm the AR staining, PCa tumor was used as a positive control. In PCa tumor AR stained the nuclei of cancer cells only. In a study to investigate EGFR expression in two groups of patients with OSCC and a control group of subjects, patients with EGFR overexpression had worse survival. ${ }^{26}$ In addition to AR protein expression, the correlation between $A R$ expression and EGFR expression was also investigated. The study identified that AR expression was positively correlated to pEGFR expression in OSCC tumors. However, no difference in tEGFR was found between ARpositive tumors and AR-negative tumors. These results showed the clue that AR may regulate EGFR phosphorylation. Based on these interesting findings, we further investigated if AR plays an important role in OSCC cells migration by regulating EGFR signaling.

In OSCC cell lines, our data identified AR expression at both the mRNA and protein levels, which were similar to a previous report. ${ }^{27}$ In the five OSCC cell lines, SCC9 cells were found to express the highest AR at both the 
mRNA and protein levels. However, neither AR mRNA nor protein was detected in SCC25 cells. So these two cell lines were selected for our current studies.

SCC9 cells treated with DHT increased migration rate concomitant with an increment of phosphorylation of EGFR and AKT. Meanwhile, SCC9 cells treated with Bicalutamide reduced migration rate, phosphorylation of EGFR and AKT. However, EGFR mRNA and tEGFR were not changed either treated with DHT or Bicalutamide. No effect was found in SCC25 cells confirming that AR promotes cell migration by activation of EGFR phosphorylation. The studies focusing on the relationship between AR and EGFR are mostly on normal prostate and prostate cancer. EGFR expression regulated by AR has been shown to be at the transcriptional level in both normal prostate and PCa cell lines. In normal prostate, this regulation is negatively regulated and in PCa is upregulated. Castrated animals treated with DHT reduced EGFR protein expression. ${ }^{28}$ As progestins and estrogens did not downregulate EGFR expression, the downregulated EGFR expression was specific to androgens. EGFR protein expression was increased in the absence of or because of reduced androgen. ${ }^{29}$ These data support that EGFR expression is downregulated by androgens in normal prostate. However, in PCa cell lines a number of studies reported that androgens upregulated EGFR. In PCa cell lines, DHT treatment enhanced EGFR mRNA levels and EGFR protein expression. ${ }^{30,31}$ In re-introducing AR into PC3 cell lines DHT treatment induced a two-fold increase in EGFR transcription and about a $50 \%$ increment of EGFR protein. EGF binding to EGFR in PC3-AR cells exposed to DHT was increased, suggesting an upregulation of EGFR expression by androgens. $^{32}$ DHT treatment also increased EGFR mRNA and protein levels in LNCaP cells. ${ }^{33}$ These studies supported that androgens upregulate EGFR expression in prostate cancer. Interestingly, in OSCC cell lines, we did not observe that AR-regulated the transcriptional level of EGFR. This finding is different from prostate or $\mathrm{PCa}$ cell lines. This difference may indicate different molecular mechanisms of $\mathrm{AR}$ in regulating EGFR between OSCC cell lines and PCa cell lines. These different molecular mechanisms deserve further investigation. Previous studies have demonstrated that in the SCC-25 cells a second smaller band which possibly corresponds to the $145 \mathrm{kDa}$ EGFR deletion mutant EGFRvIII was observed. ${ }^{27} \mathrm{~A}$ small band under tEGFR was also found in this study. DHT treatment did not change EGFRvIII expression.

In the present study, to further confirm the effect of AR on SCC9 cell migration is through targeting EGFR signaling, SCC9 cells were treated with EGF or cetuximab. The results showed that EGF increased and cetuximab suppressed SCC9 cell migration, which was similar to a previous report. ${ }^{34}$ It proved that pEGFR increased by AR in SCC9 cells is able to promote cell migration.

In conclusion, the current study identified that OSCC tumors and OSCC cells express AR. The results also proved that AR plays a pivotal role in promoting cell migration by increasing EGFR phosphorylation. Thus, our data strongly suggest that AR can be a potential target, and androgen deprivation treatment may have clinical benefits for the treatment of AR-positive OSCC patients.

\section{Acknowledgments}

This study was funded by the Medical Research Program of Chongqing Health and Family Planning Commission, Chongqing, China (2015MSXM222), and the Basic and Frontier Research Program of Yuzhong Science \& Technology Commission, Chongqing, China (No. 20160135).

\section{Disclosure}

The authors report no conflicts of interest in this work.

\section{References}

1. Bagan J, Sarrion G, Jimenez Y. Oral cancer: clinical features. Oral Oncol. 2010;46(6):414-417. doi:10.1016/j.oraloncology.2010.03.009

2. Torre LA, Siegel RL, Ward EM, Jemal A. Global cancer incidence and mortality rates and trends-an update. Cancer Epidemiol Biomarkers Prev. 2016;25(1):16-27. doi:10.1158/1055-9965.EPI-15-0578

3. Ferlay J, Soerjomataram I, Dikshit R, et al. Cancer incidence and mortality worldwide: sources, methods and major patterns in GLOBOCAN 2012. Int $J$ Cancer. 2015;136:E359-E386. doi:10.1002/ijc. 29210

4. Rivera C, Venegas B. Histological and molecular aspects of oral squamous cell carcinoma (review). Oncol Lett. 2014;8:7-11. doi:10.3892/ol.2014.2103

5. Afzali P, Ward BB. Management of the neck in oral squamous cell carcinoma: background, classification, and current philosophy. Oral Maxillofac Surg Clin North Am. 2019;31(1):69-84. doi:10.1016/j. coms.2018.09.004

6. Kramer R, Shen X, Zhou H. Tumor cell invasion and survival in head and neck cancer. Cancer Metastasis Rev. 2005;24:35-45. doi:10.1007/ s10555-005-5046-2

7. Kalyankrishna S, Grandis JR. Epidermal growth factor receptor biology in head and neck cancer. J Clin Oncol. 2006;24:2666-2672. doi:10.1200/JCO.2005.04.8306

8. Rorth P. Collective cell migration. Annu Rev Cell Dev Biol. 2009;25:407-429. doi:10.1146/annurev.cellbio.042308.113231 
9. Raben D, Baselga J, Spencer SA, et al. Radiotherapy plus cetuximab for locoregionally advanced head and neck cancer: 5-year survival data from a phase 3 randomised trial and relation between cetuximab-induced rash and survival. Lancet Oncol. 2010;11:21-28. doi:10.1016/S1470-2045(09)70311-0

10. Fung C, Grandis JR. Emerging drugs to treat squamous cell carcinomas of the head and neck. Expert Opin Emerg Drugs. 2010;15:355-373. doi:10.1517/14728214.2010.497754

11. Devlin HL, Mudryj M. Progression of prostate cancer: multiple pathways to androgen independence. Cancer Lett. 2009;274:177-186. doi:10.1016/j.canlet.2008.06.007

12. Wu TF, Luo FJ, Chang YL, et al. The oncogenic role of androgen receptors in promoting the growth of oral squamous cell carcinoma cells. Oral Dis. 2015;21(3):320-327. doi:10.1111/odi.12272

13. Chan TO, Rittenhouse SE, Tsichlis PN. AKT/PKB and other D3 phosphoinositide-regulated kinases: kinase activation by phosphoinositide-dependent phosphorylation. Annu Rev Biochem. 1999;68:965-1014. doi:10.1146/annurev.biochem.68.1.965

14. Foley J, Nickerson NK, Nam S, et al. EGFR signaling in breast cancer: bad to the bone. Semin Cell Dev Biol. 2010;21:951-960. doi:10.1016/j.semcdb.2010.08.009

15. Díaz-Serrano A, Gella P, Jiménez E, et al. Targeting EGFR in Lung Cancer: Current Standards and Developments. Drugs. 2018 jun;78 (9):893-911. doi:10.1007/s40265-018-0916-4

16. Miyamoto Y, Suyama K, Baba H. Recent advances in targeting the EGFR signaling pathway for the treatment of metastatic colorectal cancer. Int J Mol Sci. 2017;18(4):E752. doi:10.3390/ijms18040752

17. Chiang WF, Liu SY, Yen CY, et al. Association of epidermal growth factor receptor (EGFR) gene copy number amplification with neck lymph node metastasis in areca-associated oral carcinomas. Oral Oncol. 2008;44(3):270-276. doi:10.1016/j.oraloncology.2007.02.008

18. Migliaccio A, Di Domenico M, Castoria G, et al. Steroid receptor regulation of epidermal growth factor signaling through $\mathrm{Src}$ in breast and prostate cancer cells: steroid antagonist action. Cancer Res. 2005;65:10585-10593. doi:10.1158/0008-5472.CAN-05-0912

19. Zhu X, Li H, Liu JP, Funder JW. Androgen stimulates mitogen-activated protein kinase in human breast cancer cells. Mol Cell Endocrinol. 1999;152:199-206.

20. Migliaccio A, Castoria G, Di Domenico M, et al. Steroid-induced androgen receptor-oestradiol receptor beta-Src complex triggers prostate cancer cell proliferation. EMBO J. 2000;19:5406-5417. doi:10.1093/emboj/19.20.5406

21. Liang CC, Park AY, Guan JL. In vitro scratch assay: a convenient and inexpensive method for analysis of cell migration in vitro. Nat Protoc. 2007;2:329-333. doi:10.1038/nprot.2007.30

22. Pihlajamaa P, Sahu B, Jänne OA. Determinants of receptor- and tissue-specific actions in androgen signaling. Endocr Rev. 2015;36 (4):357-384. doi:10.1210/er.2015-1034
23. Ahmed A, Ali S, Sarkar FH. Advances in androgen receptor targeted therapy for prostate cancer. J Cell Physiol. 2014;229(3):271-276. doi:10.1002/jcp. 24456

24. Marocchio LS, Giudice F, Corrêa L, Pinto Junior Ddos S, de Sousa SO. Oestrogens and androgen receptors in oral squamous cell carcinoma. Acta Odontol Scand. 2013;71(6):1513-1519. doi:10.3109/00016357.2013.775335

25. Shidham VB, Komorowski RA, Machhi JK. Androgen receptor expression in metastatic adenocarcinoma in females favors a breast primary. Diagn Pathol. 2006;1:34. doi:10.1186/1746-1596-1-42

26. Costa V, Kowalski LP, Coutinho-Camillo CM, et al. EGFR amplification and expression in oral squamous cell carcinoma in young adults. Int $J$ Oral Maxillofac Surg. 2018;47(7):817-823. doi:10.1016/j.ijom.2018.01.002

27. Agostini M, Silva SD, Zecchin KG, et al. Fatty acid synthase is required for the proliferation of human oral squamous carcinoma cells. Oral Oncol. 2004;40(7):728-735. doi:10.1016/j. oraloncology.2004.01.011

28. Hammarsten P, Rudolfsson SH, Henriksson R, Wikstrom P, Bergh A. Inhibition of the epidermal growth factor receptor enhances castrationinduced prostate involution and reduces testosterone-stimulated prostate growth in adult rats. Prostate. 2007;67:573-581. doi:10.1002/pros.20529

29. St-Arnaud R, Poyet P, Walker P, Labrie F. Androgens modulate epidermal growth factor receptor levels in the rat ventral prostate. Mol Cell Endocrinol. 1988;56:21-27.

30. Liu XH, Wiley HS, Meikle AW. Androgens regulate proliferation of human prostate cancer cells in culture by increasing transforming growth factor-alpha (TGF-alpha) and epidermal growth factor (EGF)/TGF-alpha receptor. $J$ Clin Endocrinol Metab. 1993;77:1472-1478. doi:10.1210/jcem.77.6.8263129

31. Hoy JJ, Kallifatidis G, Smith DK, Lokeshwar BL. Inhibition of androgen receptor promotes CXC-chemokine receptor 7-mediated prostate cancer cell survival. Sci Rep. 2017;7(1):3058. doi:10.1038/ s41598-017-02918-3

32. Brass AL, Barnard J, Patai BL, et al. Androgen up-regulates epidermal growth factor receptor expression and binding affinity in PC3 cell lines expressing the human androgen receptor. Cancer Res. 1995;55(14):3197-3203

33. Pignon JC, Koopmansch B, Nolens G, Delacroix L, Waltregny D, Winkler R. Androgen receptor controls EGFR and ERBB2 gene expression at different levels in prostate cancer cell lines. Cancer Res. 2009;69:2941-2949. doi:10.1158/0008-5472.CAN-08-3760

34. Ohnishi Y, Yasui H, Kakudo K, Nozaki M. Regulation of cell migration via the EGFR signaling pathway in oral squamous cell carcinoma cells. Oncol Lett. 2017;13(2):930-936. doi:10.3892/ ol.2016.5500
OncoTargets and Therapy

\section{Publish your work in this journal}

OncoTargets and Therapy is an international, peer-reviewed, open access journal focusing on the pathological basis of all cancers, potential targets for therapy and treatment protocols employed to improve the management of cancer patients. The journal also focuses on the impact of management programs and new therapeutic agents and protocols on patient perspectives such as quality of life, adherence and satisfaction. The manuscript management system is completely online and includes a very quick and fair peer-review system, which is all easy to use. Visit http://www.dovepress.com/ testimonials.php to read real quotes from published authors. 